\title{
Seed Storing Potential of Some Marigold Genotypes during Storage
}

\author{
Jui Ray and Sanjoy Kumar Bordolui* \\ Department of Seed Science and Technology, Bidhan Chandra Krishi Viswavidyalaya \\ Mohanpur - 741252, Nadia, West Bengal, India \\ *Corresponding author
}

\section{A B S T R A C T}

\begin{tabular}{|l|}
\hline Ke y w o r d s \\
Marigold, \\
Deterioration and \\
storability
\end{tabular}

Marigold (Tagetes sp.) is one of the commercially exploited flower crops of both tropics and subtropics. The field trial was measured for twelve marigold genotypes in new alluvial zone at Horticulture Research station, Mondouri, B.C.K.V., Mohanpur, Nadia, West Bengal during Rabi season of 2017-18 to 2018-19.The trend in seed deterioration of those marigold genotypes was observed by storing with five treatments in seed testing laboratory, Department of Seed science and Technology, B.C.K.V. After harvesting seeds were stored in different conditions like Aluminium foil $\left(T_{1}\right)$, Cloth bag $\left(T_{2}\right)$, Polythene packet of 300 gauge $\left(T_{3}\right)$, Brown paper packet $\left(T_{4}\right)$ and refrigerator $\left(\mathrm{T}_{5}\right)$ at $8-10{ }^{\circ} \mathrm{C}$. The different seed quality parameters such as root length $(\mathrm{cm})$, shoot length $(\mathrm{cm})$, germination percentage and vigour index were recorded/determined after harvesting and at every three months interval up to twelve months of storage. Seed storage of all the genotypes failed to attain prescribed germination (70\%) as per MSCS of Marigold beyond three months after storage irrespective of the storage conditions; hence, different conditions under consideration may not be recommended for storing of Marigold seeds beyond three months.Joint consideration of germination potential and vigour index indicate that seed storage within refrigerator $\left(\mathrm{T}_{5}\right)$ as well as in 300 gauge polythene packet $\left(\mathrm{T}_{3}\right)$ may be recommended for all the genotypes studied upto three months for maintenance of its higher quality.

\section{Introduction}

Marigold is native to the New World and sacred flowers of the Aztecs, journeyed across the Atlantic Ocean twice to travel 3,000 miles north of their centre of origin. During 16th century, Portuguese introduced Marigold in India (Gawle et al., 2012). The flower ranks first among the commercial loose flowers in India followed by chrysanthemum, jasmine, tuberose, crossandra and barleria (Gajanana and Sudha, 2006, Chakraborty et al., 2019).
In India 511.31 thousand MT marigold was produced under an area of 55.89 thousand hectare (Saxena and Gandhi, 2015).

West Bengal has become one of the leading states in marigold commercial cultivation across the country. Especially, African Marigold (Tagetes erecta) is grown at a large scale mainly in the districts of Midnapore, Howrah, Hooghly, 24 Parganas and Nadia. Though works have done to improve flower quality parameters in marigold, but the 
successful establishment of the crop typically depends upon good quality seeds. Viability and vigour of the seeds are regulated by many physico-chemical factors (Kumari et al., 2017). Seeds of marigold also consistently loose its viability and vigour with the advancement of storage due to several factors $v i z$, moisture content of the seed, atmospheric humidity, temperature, initial seed quality, physical and chemical composition of seed, gaseous exchange, storage structure and packaging materials (Mahato et al., 2019). Therefore, maintenance of higher germinability of carry over seed till next sowing season is of vital importance to seed producers, which can be made possible through storing the seeds under controlled conditions i.e., combination of low temperature and relative humidity (Selvaraju et al., 1999 and Kumar et al., 2014). But storing the seeds in controlled conditions is practically very difficult in developing countries like India due to higher cost.

Keeping all the facts mentioned above in mind present research work was conducted in seed testing laboratory, Department of Seed science and Technology, Bidhan Chandra KrishiViswavidyalaya, Mohanpur, Nadia, West Bengal, India, during 2018, to identify proper seed storage condition(s) for maintenance of high seed vigour and viability.

\section{Materials and Methods}

The laboratory experiment was carried out in seed testing laboratory, Department of Seed science and Technology, Bidhan Chandra KrishiViswavidyalaya, Mohanpur, Nadia, West Bengal, India, during 2018. Immediately after harvesting, seeds were properly sun dried to its $6 \%$ moisture content (approximately)of flowers collected from control plot of individual genotype for each replication were kept in four different packaging materials and one condition viz.,
Aluminium foil $\left(\mathrm{T}_{1}\right)$, Cloth bag $\left(\mathrm{T}_{2}\right)$, Polythene packet $\left(\mathrm{T}_{3}\right)$ of 300 gauge thickness and Brown paper packet $\left(\mathrm{T}_{4}\right)$ as well as within refrigerator $\left(\mathrm{T}_{5}\right)$ at around $8-10{ }^{0} \mathrm{C}$ (Chakraborty et al., 2020). Different seed quality parameters such as root length $(\mathrm{cm})$, shoot length $(\mathrm{cm})$, seedling length $(\mathrm{cm})$, germination percentage and vigour index were recorded/determined at pre-storage condition as well as at every three months interval up to one year of storage.

\section{Results and Discussion}

All the quality parameters studied were found to be significant for harvest fresh seeds excepting seedling shoot length. When testing was made for stored seeds at five different storage intervals all the parameters varied significantly among the genotypes, influence of different storage conditions as well as its interactions with the exception of seedling shoot length for storage conditions only at both nine and twelve months of storage.

Significant variation among the genotypes was noted for all the physiological parameters studied excepting shoot length of seedlings. Highest average germination percentage of harvest fresh seeds was recorded for $V_{9}$ (88.67) followed by $V_{2}, V_{8}, V_{6}, V_{5}$ and $V_{3}$, though non-significant difference could be noticed among these five genotypes for this important seed parameter, while it was lowest for both $\mathrm{V}_{10}$ (83.54) and $\mathrm{V}_{11}$ (83.89). Significantly longest seedling root was found to be produced by $\mathrm{V}_{9}, \mathrm{~V}_{10}$ and $\mathrm{V}_{5}$, while it was shortest for both $\mathrm{V}_{7}$ and $\mathrm{V}_{11}$. Though non-significant, longest and shortest seedling shoot was noticed for $\mathrm{V}_{9}$ and $\mathrm{V}_{5}$ respectively. Significant diversity among genotypes could, therefore, be recognised through seedling root length. While considering vigour index, one of the most vital seed quality parameters, it is to be noted that a wide range of vigour index was determined for the genotypes as 620.32 
(for $V_{7}$ ) to 919.27 (for $V_{9}$ ). Higher vigour index of seeds determined for $\mathrm{V}_{9}, \mathrm{~V}_{5}, \mathrm{~V}_{12}$ and $\mathrm{V}_{10}$ may lead to recommend for its better commercial utilization through concomitant consideration of its higher germination potential (Fig. 1-10 and Table 1-5).

Germination percentage at three, six, nine and twelve months after storage

While considering the average performance of the genotypes after three months of storage, variation in the germination potential was found to be significant and maintained within the minimum seed certification standard (MSCS) specified for Marigold, though a great reduction was noticed from pre-storage condition irrespective of the genotypes. It was of maximum value for $\mathrm{V}_{9}$ (Pusa Narangi) followed by $V_{12}, V_{8}$ and $V_{2}$, performance of $\mathrm{V}_{12}, \quad \mathrm{~V}_{8}$ and $\mathrm{V}_{2}$ was observed to be statistically at per with each other and it was of lowest value for $\mathrm{V}_{10}$, which was noted to be almost at per with the value prescribed in MSCS (Table 2). Refrigerator storage exerted significantly highest influence average over genotypes in comparison to that of other storage conditions and it was followed by polythene packet, aluminium foil, brown paper packet and cloth bag. While analysis was made for seed storage of individual genotypes under different storage conditions, almost similar trend could be noticed as could be revealed for average influence of the storage conditions; slight deviation could be noticed for $\mathrm{V}_{6}$ only, for which influence of aluminium foil and brown paper packet was noticed to be significantly at per with each other.

Consistent reduction in average germination potential at individual genotypes could be revealed through Table 2, and it attained the lower magnitude than the prescribed value under MSCS on six months storage and onwards irrespective of the storage conditions. The trend in performance of $\mathrm{V}_{9}$ was best at six months after storage followed by that of $\mathrm{V}_{12}, \mathrm{~V}_{8}, \mathrm{~V}_{5}$ and $\mathrm{V}_{3}$; it was also of minimum value for $\mathrm{V}_{10}$. Ranking of individual storage conditions maintained exactly same as was noticed after three months of storage. Similar trend in response of individual genotypes towards different storage conditions was noticed after six months of storage for this important parameter. $\mathrm{V}_{9}$ secured the same top rank for its germination potential at both nine and twelve months of storage. It was followed by $\mathrm{V}_{12}, \mathrm{~V}_{8}$ and $\mathrm{V}_{5}$ and after both nine and twelve months of storage, significantly similar performance of $\mathrm{V}_{8}$ and $\mathrm{V}_{5}$ was noticed. Performance of $\mathrm{V}_{10}$ was consistently lowest at different stages of storage duration. Position of other genotypes with regard to its germination potential was found to be shuffled at different stages of storage durations, may be due to preference towards the unique characteristics of the storage condition. Combinations of genotypes and storage conditions exhibited similar trend in response in all storages of storage durations including nine and twelve months of storage, slight deviation could be noticed for $V_{6}$ and $\mathrm{V}_{9}$ at nine months of storage and $\mathrm{V}_{5}, \mathrm{~V}_{8}, \mathrm{~V}_{9}$ and $\mathrm{V}_{10}$ at twelve months of storage. Considering the prescribed germination percentage under MSCS of Marigold vis-à-vis the experimental findings recorded here in for this important parameter, recommendation can be made for safe storage till three months of storage under the storage conditions included in the present investigation.

\section{Seedling root length $(\mathrm{cm})$ at three, six, nine and twelve months after storage}

Root length of seedlings at ten days of setting was as maximum as $6.25 \mathrm{~cm}$ for $\mathrm{V}_{9}$ followed by $\mathrm{V}_{5}, \mathrm{~V}_{6}, \mathrm{~V}_{7}$ and $\mathrm{V}_{4}$, statistical similarity could be noticed between $\mathrm{V}_{5}$ and $\mathrm{V}_{6}$ as well as between $\mathrm{V}_{7}$ and $\mathrm{V}_{4}$; it was of shortest 
length for $\mathrm{V}_{11}$ after three months of storage when average was made over the storage conditions. Refrigerated stored seeds became able to produce seedlings with longest root length followed by that stored in cloth bag, brown paper packet, polythene packet and aluminium foil, when average was made over the genotypes; non-significant difference could be noticed among refrigerated storage, cloth bag and brown paper storage as well as between polythene packet and aluminium foil storage.

While considering the performance of individual genotypes stored under different conditions, best response towards refrigerated storage could be noticed for seven numbers of genotypes, the deviation could be noticed for $\mathrm{V}_{1}, \mathrm{~V}_{2}, \mathrm{~V}_{3}, \mathrm{~V}_{4}$ and $\mathrm{V}_{6}$. The trend recorded for individual genotypes for production of seedling root could be ascertained in a very erratic manner indicating different mode of response of the genotypes for expression of this character particularly at the stage of three months of storage.

Average seedling root length of $\mathrm{V}_{9}$ was found to be maximum followed by $\mathrm{V}_{5}, \mathrm{~V}_{6}, \mathrm{~V}_{3}, \mathrm{~V}_{4}$ and $V_{7}$ at six months of storage, statistical similarity between $\mathrm{V}_{5}$ and $\mathrm{V}_{6}$ as well as between $V_{4}$ and $V_{7}$ could be noticed. Though varied in magnitude, longer root length of seedlings could be noticed for all other storage conditions over that kept in aluminium foil and statistical similarity amongst those conditions could be noticed. Similar to the three months of storage duration, it becomes difficult to ascertain the performance of individual genotypes towards storage condition for a definite trend; some genotypes responded better under refrigerated condition, some under brown paper, a few under polythene packet.

The same genotype $\mathrm{V}_{9}$ produced maximum seedling root length at both nine and twelve months of storage, when average was made over genotypes and it became statistically same with that of $V_{6} . V_{5}$ and $V_{3}$ occupied the second and third position respectively at both of these storage durations and it was of significantly lowest magnitude for $V_{11}$. Significantly maximum seedling root length was recorded after refrigerator storage on both the durations, while it was significantly similar to that of cloth bag and polythene packet at nine months of storage and statistically at per values could be regarded for storage in cloth bag and polythene packet on an average after twelve months of storage.

Tremendous erratic behaviour in trend could be noticed for production of seedling root length at both nine and twelve months of storage particularly for longest and shortest length. It becomes very difficult to clarify the situations under which response of individual genotypes towards different storage conditions for expression of this specific character became highly erratic as expressed by either enhancement or reduction or both in seedling root length with the advancement in storage duration.

Seedling shoots length $(\mathrm{cm})$ at three, six, nine and twelve months after storage

Clarification on variation in seedling shoot length as recorded for the average genotypic performance, mean influence of storage conditions as well as its interaction may throw an aberration in normal scenario as could be expected for different storage durations. Seedling shoot length recorded to be maximum for $\mathrm{V}_{12}$ on an average, irrespective of the storage duration and it was of significantly lowest magnitude for $\mathrm{V}_{11}$; position of other genotypes tremendously varied in different storage durations viz., $\mathrm{V}_{11}$ and $\mathrm{V}_{1}$ occupied the lowest position at three months after storage; $\mathrm{V}_{11}, \mathrm{~V}_{6}, \mathrm{~V}_{5}$ maintained significantly the same position at six months 
after storage; $\mathrm{V}_{11}, \mathrm{~V}_{4}, \mathrm{~V}_{5}, \mathrm{~V}_{3}$ and $\mathrm{V}_{8}$ were in the same position at twelve months of storage; and peculiar situation was noticed at nine months of storage, wherein eight out of twelve genotypes significantly occupied the same position excepting $\mathrm{V}_{2}, \mathrm{~V}_{7}, \mathrm{~V}_{10}$ and $\mathrm{V}_{12}$.

Consideration of average influence of these storage conditions directed to select refrigerator storage as the best one for exhibiting longest seedling shoot length till nine months of storage over other storage conditions, non-significant difference could be noticed among the influence of storage conditions at twelve months of storage. Response of individual genotypes towards storage conditions for longest seedling shoot length production varied over the storage durations: $\mathrm{V}_{1}, \mathrm{~V}_{4}$ and $\mathrm{V}_{5}$ responded in a better way towards storage in aluminium foil $\left(T_{1}\right)$, $\mathrm{V}_{2}, \mathrm{~V}_{3}, \mathrm{~V}_{6}, \mathrm{~V}_{7}, \mathrm{~V}_{8}, \mathrm{~V}_{11}$ and $\mathrm{V}_{12}$ towards refrigerated storage $\left(T_{5}\right)$, and $V_{10}$ and $V_{9}$ towards polythene packet $\left(\mathrm{T}_{3}\right)$ and brown paper packet $\left(\mathrm{T}_{4}\right)$ respectively at three months of storage; the genotypes responded in very haphazard manner at six months storage and onwards towards storage conditions, even non-significant difference could be clearly marked in response of some genotypes towards storage conditions indicating genotype specific response for expression of this parameter.

Table.1 Germination percentage, root length $(\mathrm{cm})$, shoot length $(\mathrm{cm})$ and vigour index of harvest fresh seeds of different Marigold genotypes

\begin{tabular}{|c|c|c|c|c|c|}
\hline SI No. & Genotypes & $\begin{array}{c}\text { Germination } \\
(\%)\end{array}$ & $\begin{array}{l}\text { Root length } \\
\quad(\mathrm{cm})\end{array}$ & $\begin{array}{l}\text { Shoot length } \\
\text { (cm) }\end{array}$ & Vigour index \\
\hline 1. & $\mathbf{V}_{1}$ & $67.61(85.52)$ & 5.07 & 3.40 & 723.96 \\
\hline 2. & $\mathbf{V}_{2}$ & $69.46(87.71)$ & 5.03 & 3.40 & 739.62 \\
\hline 3. & $\mathbf{V}_{3}$ & $69.01(87.19)$ & 4.47 & 3.37 & 682.99 \\
\hline 4. & $\mathbf{V}_{4}$ & $68.53(86.63)$ & 5.70 & 3.43 & 791.16 \\
\hline 5. & $V_{5}$ & $69.27(87.49)$ & 6.57 & 3.17 & 851.49 \\
\hline 6. & $V_{6}$ & $69.33(87.57)$ & 4.80 & 3.43 & 720.94 \\
\hline 7. & $\mathbf{V}_{7}$ & $68.48(86.57)$ & 3.83 & 3.33 & 620.32 \\
\hline 8. & $\mathbf{V}_{8}$ & $69.41(87.65)$ & 4.93 & 3.47 & 736.30 \\
\hline 9. & $V_{9}$ & $70.31(88.67)$ & 6.70 & 3.67 & 919.27 \\
\hline 10. & $\mathbf{V}_{10}$ & $66.05(83.54)$ & 6.60 & 3.30 & 827.08 \\
\hline 11. & $V_{11}$ & $66.33(83.89)$ & 4.37 & 3.40 & 651.53 \\
\hline \multirow[t]{3}{*}{12.} & $\mathrm{~V}_{12}$ & $68.67(86.80)$ & 6.03 & 3.57 & 833.33 \\
\hline & $\operatorname{SEm}( \pm)$ & 0.49 & 0.20 & 0.15 & 8.08 \\
\hline & $\operatorname{LSD}(0.05)$ & 1.44 & 0.57 & NS & 23.72 \\
\hline
\end{tabular}

$\mathrm{V}_{1}=$ Bidhan Marigold-1, $\mathrm{V}_{2}=$ Bidhan Basanti, $\mathrm{V}_{3}=\mathrm{BM}-3, \mathrm{~V}_{4}=\mathrm{BM}-4, \mathrm{~V}_{5}=\mathrm{BM}-5, \mathrm{~V}_{6}=\mathrm{BM}-6, \mathrm{~V}_{7}=$ Arka Bangara$1, \mathrm{~V}_{8}=$ Arka Bangara-2, $\mathrm{V}_{9}=$ Pusa Narangi, $\mathrm{V}_{10}=$ Yellow Single, $\mathrm{V}_{11}=$ Orange Single, $\mathrm{V}_{12}=$ Red Fresh Inka 
Table.2 Germination percentage of different Marigold genotypes at three, six, nine and twelve months after storage

\begin{tabular}{|c|c|c|c|c|c|}
\hline \multirow{2}{*}{$\begin{array}{c}\text { Sl. } \\
\text { No. }\end{array}$} & \multirow{2}{*}{$\begin{array}{c}\text { Treatment } \\
\text { X } \\
\text { Genotype }\end{array}$} & \multicolumn{4}{|c|}{ Germination \% } \\
\hline & & Three month & Six month & Nine month & Twelve month \\
\hline 1. & $\mathbf{T}_{1} \mathbf{V}_{1}$ & $72.25(58.19)$ & $63.26(52.67)$ & $52.26(46.28)$ & $44.21(41.66)$ \\
\hline 2. & $\mathbf{T}_{1} \mathbf{V}_{2}$ & $74.56(59.68)$ & $65.48(54.10)$ & $54.42(47.52)$ & $46.38(42.91)$ \\
\hline 3. & $\mathbf{T}_{1} \mathbf{V}_{3}$ & $74.25(59.48)$ & $65.90(54.25)$ & $54.82(47.75)$ & $46.73(43.11)$ \\
\hline 4. & $\mathbf{T}_{1} \mathbf{V}_{4}$ & $73.72(59.14)$ & $65.51(54.01)$ & $54.33(47.46)$ & $46.36(42.89)$ \\
\hline 5. & $\mathbf{T}_{1} \mathbf{V}_{5}$ & $74.37(59.56)$ & $66.05(54.34)$ & $55.02(47.86)$ & $46.90(43.21)$ \\
\hline 6. & $\mathbf{T}_{1} \mathbf{V}_{6}$ & $74.18(59.44)$ & $65.80(54.19)$ & $54.50(47.56)$ & $46.61(43.04)$ \\
\hline 7. & $\mathbf{T}_{1} \mathbf{V}_{7}$ & $74.15(59.42)$ & $65.82(54.20)$ & $54.40(47.51)$ & $46.34(42.88)$ \\
\hline 8. & $\mathbf{T}_{1} \mathbf{V}_{8}$ & $74.74(59.80)$ & $66.40(54.55)$ & $55.54(48.16)$ & $47.62(43.62)$ \\
\hline 9. & $\mathbf{T}_{1} \mathbf{V}_{9}$ & $75.53(60.33)$ & $67.39(55.15)$ & $56.28(48.59)$ & $48.30(44.01)$ \\
\hline 10. & $\mathbf{T}_{1} \mathbf{V}_{10}$ & $69.88(56.69)$ & $61.61(51.69)$ & $50.76(45.42)$ & $43.44(41.21)$ \\
\hline 11. & $\mathbf{T}_{1} \mathbf{V}_{11}$ & $71.29(57.58)$ & $62.96(52.49)$ & $51.78(46.00)$ & $43.81(41.43)$ \\
\hline 12. & $T_{1} V_{12}$ & $74.81(59.85)$ & $66.52(54.62)$ & $55.76(48.29)$ & $47.82(43.73)$ \\
\hline 13. & $\mathbf{T}_{2} \mathbf{V}_{1}$ & $71.17(57.50)$ & $62.15(52.01)$ & $51.16(45.65)$ & $43.13(41.04)$ \\
\hline 14. & $\mathbf{T}_{2} \mathbf{V}_{2}$ & $73.76(59.16)$ & $64.38(53.33)$ & $53.35(46.90)$ & $45.34(42.31)$ \\
\hline 15. & $\mathbf{T}_{2} \mathbf{V}_{3}$ & $73.25(58.83)$ & $64.69(53.52)$ & $53.71(47.11)$ & $45.69(42.51)$ \\
\hline 16. & $\mathbf{T}_{2} \mathbf{V}_{4}$ & $72.72(58.49)$ & $64.15(53.20)$ & $53.57(47.03)$ & $45.47(42.39)$ \\
\hline 17. & $\mathbf{T}_{2} \mathbf{V}_{5}$ & $73.73(59.15)$ & $65.26(53.86)$ & $54.66(47.65)$ & $46.54(43.10)$ \\
\hline 18. & $\mathbf{T}_{2} \mathbf{V}_{6}$ & $73.27(58.84)$ & $64.73(53.55)$ & $53.69(47.10)$ & $45.64(42.48)$ \\
\hline 19. & $\mathbf{T}_{2} \mathbf{V}_{7}$ & $73.25(58.83)$ & $64.83(53.60)$ & $53.27(46.86)$ & $45.28(42.27)$ \\
\hline 20. & $\mathbf{T}_{2} \mathbf{V}_{8}$ & $73.54(59.02)$ & $65.23(53.85)$ & $54.34(47.47)$ & $46.34(42.88)$ \\
\hline 21. & $\mathbf{T}_{2} \mathbf{V}_{9}$ & $74.27(59.50)$ & $65.97(54.29)$ & $55.94(48.39)$ & $47.85(43.75)$ \\
\hline 22. & $\mathbf{T}_{2} \mathbf{V}_{10}$ & $68.79(56.01)$ & $60.46(51.02)$ & $49.67(44.79)$ & $41.73(40.22)$ \\
\hline 23. & $\mathbf{T}_{2} \mathbf{V}_{11}$ & $70.38(57.00)$ & $61.93(51.88)$ & $50.71(45.39)$ & $42.69(40.78)$ \\
\hline 24. & $\mathbf{T}_{2} \mathbf{V}_{12}$ & $73.37(58.91)$ & $65.25(53.86)$ & $54.77(47.72)$ & $46.66(43.07)$ \\
\hline 25. & $\mathbf{T}_{3} \mathbf{V}_{1}$ & $72.86(58.58)$ & $63.87(53.03)$ & $52.83(46.60)$ & $44.82(42.01)$ \\
\hline 26. & $\mathbf{T}_{3} \mathbf{V}_{2}$ & $75.04(60.01)$ & $66.22(54.45)$ & $55.23(47.98)$ & $47.21(43.38)$ \\
\hline 27. & $\mathbf{T}_{\mathbf{3}} \mathbf{V}_{\mathbf{3}}$ & 74.84 (59.87) & $66.71(54.74)$ & $55.77(48.29)$ & $47.87(43.76)$ \\
\hline 28. & $\mathbf{T}_{3} \mathbf{V}_{4}$ & $74.10(59.38)$ & $65.92(54.26)$ & $54.66(47.65)$ & $46.65(43.06)$ \\
\hline 29. & $\mathbf{T}_{3} \mathbf{V}_{5}$ & $74.83(59.86)$ & $66.70(54.73)$ & $55.72(48.27)$ & $47.69(43.66)$ \\
\hline 30. & $T_{3} V_{6}$ & $74.71(59.79)$ & $66.54(54.64)$ & $55.41(48.09)$ & $47.39(43.49)$ \\
\hline 31. & $\mathbf{T}_{3} \mathbf{V}_{7}$ & $74.31(59.52)$ & $66.17(54.41)$ & $55.24(47.99)$ & $47.17(43.36)$ \\
\hline 32. & $\mathbf{T}_{3} \mathbf{V}_{8}$ & $75.14(60.07)$ & $66.97(54.90)$ & $55.72(48.27)$ & $47.81(43.73)$ \\
\hline 33. & $\mathbf{T}_{3} \mathbf{V}_{9}$ & $76.24(60.80)$ & $68.20(55.65)$ & $57.20(49.12)$ & $49.25(44.55)$ \\
\hline 34. & $\mathbf{T}_{3} \mathbf{V}_{10}$ & $70.73(57.23)$ & $62.53(52.24)$ & $51.43(45.80)$ & $43.44(41.21)$ \\
\hline 35. & $\mathbf{T}_{3} \mathbf{V}_{11}$ & $71.86(57.94)$ & $63.65(52.90)$ & $52.70(46.53)$ & $44.74(41.96)$ \\
\hline 36. & $\mathbf{T}_{3} \mathbf{V}_{12}$ & $75.28(60.16)$ & $67.13(55.10)$ & $56.41(48.66)$ & $48.43(44.08)$ \\
\hline 37. & $\mathbf{T}_{4} \mathbf{V}_{1}$ & $71.77(57.88)$ & $62.77(52.38)$ & $51.74(45.98)$ & $43.72(41.37)$ \\
\hline
\end{tabular}




\begin{tabular}{|c|c|c|c|c|c|}
\hline 38. & $\mathbf{T}_{4} \mathbf{V}_{2}$ & $73.98(59.30)$ & $64.86(53.62)$ & $53.84(47.18)$ & $45.80(42.57)$ \\
\hline 39. & $\mathbf{T}_{4} \mathbf{V}_{3}$ & $73.85(59.22)$ & $65.51(54.01)$ & $54.46(47.54)$ & $46.46(42.95)$ \\
\hline 40. & $\mathbf{T}_{4} \mathbf{V}_{4}$ & 73.09 (58.73) & $64.87(53.63)$ & $53.81(47.17)$ & $45.80(42.57)$ \\
\hline 41. & $\mathbf{T}_{4} \mathbf{V}_{5}$ & $74.02(59.33)$ & 65.69 (54.12) & $54.86(47.77)$ & $46.79(43.14)$ \\
\hline 42. & $\mathbf{T}_{4} \mathbf{V}_{6}$ & $74.21(59.46)$ & $65.50(54.01)$ & $54.36(47.48)$ & $46.22(42.82)$ \\
\hline 43. & $\mathbf{T}_{4} \mathbf{V}_{7}$ & $73.91(59.26)$ & $65.58(54.05)$ & $54.20(47.39)$ & $45.97(42.67)$ \\
\hline 44. & $\mathbf{T}_{4} \mathbf{V}_{8}$ & $74.23(59.47)$ & $65.90(54.25)$ & $54.95(47.82)$ & $46.87(43.19)$ \\
\hline 45. & $\mathbf{T}_{4} \mathbf{V}_{9}$ & $74.81(59.85)$ & $66.67(54.72)$ & $56.02(48.44)$ & $48.07(43.88)$ \\
\hline 46. & $T_{4} V_{10}$ & 69.25 (56.30) & $60.99(51.33)$ & 49.87 (44.91) & $41.92(40.34)$ \\
\hline 47. & $\mathbf{T}_{4} \mathbf{V}_{11}$ & 70.89 (57.33) & $62.56(52.25)$ & $51.57(45.88)$ & 43.47 (41.23) \\
\hline 48. & $T_{4} V_{12}$ & 74.34 (59.54) & 66.05 (54.34) & $55.31(48.03)$ & 47.35 (43.46) \\
\hline 49. & $\mathbf{T}_{5} \mathbf{V}_{1}$ & 73.77 (59.17) & $64.74(53.55)$ & $53.72(47.11)$ & $45.68(42.50)$ \\
\hline 50. & $\mathbf{T}_{5} \mathbf{V}_{2}$ & $75.78(60.50)$ & $66.83(54.81)$ & $55.82(48.32)$ & $47.81(43.73)$ \\
\hline 51. & $\mathbf{T}_{5} \mathbf{V}_{\mathbf{3}}$ & $75.22(60.12)$ & $66.97(54.90)$ & $56.16(48.52)$ & $48.30(44.01)$ \\
\hline 52. & $\mathbf{T}_{5} \mathbf{V}_{4}$ & $74.76(59.82)$ & $66.50(54.61)$ & $55.46(48.12)$ & $47.42(43.51)$ \\
\hline 53. & $T_{5} V_{5}$ & $75.56(60.35)$ & $67.30(55.10)$ & $56.34(48.62)$ & 48.59 (44.17) \\
\hline 54. & $\mathbf{T}_{5} \mathbf{V}_{6}$ & $75.38(60.23)$ & 67.05 (54.95) & $56.03(48.44)$ & $48.02(43.85)$ \\
\hline 55. & $\mathbf{T}_{5} \mathbf{V}_{7}$ & $74.72(59.79)$ & $66.38(54.54)$ & $55.52(48.15)$ & 47.55 \\
\hline 56. & $\mathbf{T}_{5} \mathbf{V}_{8}$ & $75.66(60.41)$ & $67.47(55.21)$ & $56.13(48.50)$ & $48.21(43.96)$ \\
\hline 57. & $\mathbf{T}_{5} \mathbf{V}_{9}$ & 76.79 (61.18) & $68.80(56.02)$ & $57.85(49.50)$ & $49.88(44.91)$ \\
\hline 58. & $T_{5} V_{10}$ & 71.55 (57.74) & $63.17(52.62)$ & $52.32(46.31)$ & $44.47(41.81)$ \\
\hline 59. & $\mathbf{T}_{5} \mathbf{V}_{11}$ & $72.35(58.25)$ & $64.07(53.15)$ & $53.11(46.76)$ & $45.23(42.25)$ \\
\hline 60. & $\mathbf{T}_{5} \mathbf{V}_{12}$ & $75.72(60.46)$ & $67.51(55.23)$ & $56.81(48.89)$ & $48.86(44.33)$ \\
\hline \multirow{2}{*}{\multicolumn{2}{|c|}{$\begin{array}{l}\operatorname{SEm}( \pm) \\
\operatorname{LSD}(0.05)\end{array}$}} & 0.052 & 0.034 & 0.027 & 0.054 \\
\hline & & 0.146 & 0.094 & 0.075 & 0.152 \\
\hline
\end{tabular}

Note: $\mathrm{T}_{1}=$ Aluminium foil, $\mathrm{T}_{2}=$ Cloth bag, $\mathrm{T}_{3}=$ Polythene packet, $\mathrm{T}_{4}=$ Brown paper packet, $\mathrm{T}_{5}=$ Freeze, $\mathrm{V}_{1}=$ Bidhan Marigold-1, $\mathrm{V}_{2}=$ Bidhan Basanti, $\mathrm{V}_{3}=\mathrm{BM}-3, \mathrm{~V}_{4}=\mathrm{BM}-4, \mathrm{~V}_{5}=\mathrm{BM}-5, \mathrm{~V}_{6}=\mathrm{BM}-6, \mathrm{~V}_{7}=$ Arka Bangara $-1, \mathrm{~V}_{8}=$ Arka Bangara $-2, \mathrm{~V}_{9}=$ Pusa Narangi, $\mathrm{V}_{10}=$ Yellow Single, $\mathrm{V}_{11}=$ Orange Single, $\mathrm{V}_{12}=$ Red Fresh Inka 
Table.3 Seedling root length $(\mathrm{cm})$ of different Marigold genotypes at three, six, nine and twelve monthsafter storage

\begin{tabular}{|c|c|c|c|c|c|}
\hline \multirow{2}{*}{$\begin{array}{c}\text { Sl. } \\
\text { No. }\end{array}$} & \multirow{2}{*}{$\begin{array}{c}\text { Treatment } \\
\text { X } \\
\text { Genotype }\end{array}$} & \multicolumn{4}{|c|}{ Seedling root length $(\mathbf{c m})$} \\
\hline & & Three month & Six month & Nine month & Twelve month \\
\hline 1. & $\mathbf{T}_{1} \mathbf{V}_{1}$ & 3.93 & 3.37 & 3.13 & 3.13 \\
\hline 2. & $\mathbf{T}_{1} \mathbf{V}_{2}$ & 4.23 & 3.40 & 3.30 & 3.07 \\
\hline 3. & $\mathbf{T}_{1} \mathbf{V}_{3}$ & 4.40 & 4.03 & 3.63 & 2.97 \\
\hline 4. & $\mathbf{T}_{1} \mathbf{V}_{4}$ & 4.37 & 3.50 & 3.07 & 3.03 \\
\hline 5. & $\mathbf{T}_{1} \mathbf{V}_{5}$ & 4.07 & 3.67 & 3.23 & 3.10 \\
\hline 6. & $T_{1} V_{6}$ & 3.80 & 3.57 & 3.27 & 3.10 \\
\hline 7. & $\mathbf{T}_{1} \mathbf{V}_{7}$ & 5.00 & 4.27 & 3.87 & 3.27 \\
\hline 8. & $\mathbf{T}_{1} \mathbf{V}_{8}$ & 5.20 & 3.87 & 3.37 & 3.07 \\
\hline 9. & $T_{1} V_{9}$ & 5.67 & 5.27 & 4.87 & 4.33 \\
\hline 10. & $\mathbf{T}_{1} \mathbf{V}_{10}$ & 4.33 & 4.23 & 3.87 & 3.30 \\
\hline 11. & $\mathbf{T}_{1} \mathbf{V}_{11}$ & 4.63 & 3.83 & 3.50 & 3.20 \\
\hline 12. & $\mathbf{T}_{1} \mathbf{V}_{12}$ & 4.53 & 4.27 & 3.83 & 3.30 \\
\hline 13. & $\mathbf{T}_{2} \mathbf{V}_{1}$ & 5.73 & 4.80 & 4.13 & 3.43 \\
\hline 14. & $\mathbf{T}_{2} \mathbf{V}_{2}$ & 5.30 & 4.50 & 4.03 & 4.47 \\
\hline 15. & $\mathbf{T}_{2} \mathbf{V}_{3}$ & 6.60 & 5.63 & 5.07 & 4.37 \\
\hline 16. & $\mathbf{T}_{2} \mathbf{V}_{4}$ & 6.73 & 6.03 & 5.40 & 4.40 \\
\hline 17. & $\mathbf{T}_{2} \mathbf{V}_{5}$ & 6.43 & 5.90 & 5.30 & 4.87 \\
\hline 18. & $T_{2} V_{6}$ & 7.07 & 6.23 & 5.40 & 4.73 \\
\hline 19. & $\mathbf{T}_{2} \mathbf{V}_{7}$ & 5.63 & 5.27 & 4.83 & 4.37 \\
\hline 20. & $\mathbf{T}_{2} \mathbf{V}_{8}$ & 5.83 & 4.83 & 4.30 & 3.77 \\
\hline 21. & $\mathbf{T}_{2} \mathbf{V}_{9}$ & 6.47 & 5.77 & 5.07 & 4.73 \\
\hline 22. & $\mathbf{T}_{2} \mathbf{V}_{10}$ & 5.57 & 4.73 & 4.23 & 3.83 \\
\hline 23. & $\mathbf{T}_{2} \mathbf{V}_{11}$ & 3.17 & 3.17 & 3.13 & 3.07 \\
\hline 24. & $\mathbf{T}_{2} \mathbf{V}_{12}$ & 3.57 & 3.23 & 3.07 & 3.00 \\
\hline 25. & $\mathbf{T}_{3} \mathbf{V}_{1}$ & 5.23 & 4.43 & 3.67 & 3.27 \\
\hline 26. & $\mathbf{T}_{3} \mathbf{V}_{2}$ & 5.37 & 4.30 & 4.53 & 3.10 \\
\hline 27. & $\mathbf{T}_{3} \mathbf{V}_{\mathbf{3}}$ & 5.67 & 5.40 & 4.53 & 3.83 \\
\hline 28. & $\mathbf{T}_{3} \mathbf{V}_{4}$ & 5.27 & 5.10 & 4.57 & 3.53 \\
\hline 29. & $\mathbf{T}_{3} \mathbf{V}_{5}$ & 6.17 & 5.63 & 5.10 & 4.70 \\
\hline 30. & $\mathbf{T}_{3} \mathbf{V}_{6}$ & 5.83 & 5.27 & 4.70 & 5.87 \\
\hline 31. & $\mathbf{T}_{3} \mathbf{V}_{7}$ & 6.33 & 5.80 & 5.10 & 3.63 \\
\hline 32. & $\mathbf{T}_{3} \mathbf{V}_{8}$ & 5.80 & 5.23 & 4.77 & 3.57 \\
\hline 33. & $\mathbf{T}_{3} \mathbf{V}_{9}$ & 5.77 & 4.87 & 4.33 & 4.17 \\
\hline 34. & $\mathbf{T}_{3} \mathbf{V}_{10}$ & 5.40 & 4.70 & 4.27 & 3.83 \\
\hline 35. & $\mathbf{T}_{3} \mathbf{V}_{11}$ & 4.63 & 4.43 & 3.87 & 3.67 \\
\hline 36. & $\mathbf{T}_{3} \mathbf{V}_{12}$ & 5.67 & 4.93 & 4.47 & 4.83 \\
\hline 37. & $\mathbf{T}_{4} \mathbf{V}_{1}$ & 5.40 & 4.77 & 4.47 & 4.27 \\
\hline
\end{tabular}




\begin{tabular}{|c|c|c|c|c|c|}
\hline 38. & $\mathbf{T}_{4} \mathbf{V}_{2}$ & 5.97 & 5.07 & 4.43 & 3.83 \\
\hline 39. & $\mathbf{T}_{\mathbf{4}} \mathbf{V}_{\mathbf{3}}$ & 5.70 & 5.33 & 4.93 & 4.47 \\
\hline 40. & $\mathbf{T}_{4} \mathbf{V}_{4}$ & 6.07 & 5.37 & 4.87 & 4.30 \\
\hline 41. & $\mathbf{T}_{4} \mathbf{V}_{5}$ & 6.30 & 5.53 & 4.63 & 4.10 \\
\hline 42. & $\mathbf{T}_{4} \mathbf{V}_{6}$ & 5.90 & 5.13 & 4.53 & 3.87 \\
\hline 43. & $\mathbf{T}_{4} \mathbf{V}_{7}$ & 5.57 & 3.60 & 3.03 & 3.10 \\
\hline 44. & $\mathbf{T}_{4} \mathbf{V}_{8}$ & 4.63 & 3.57 & 3.27 & 3.07 \\
\hline 45. & $\mathbf{T}_{4} \mathbf{V}_{9}$ & 6.80 & 6.13 & 5.83 & 5.13 \\
\hline 46. & $\mathbf{T}_{4} \mathbf{V}_{10}$ & 5.20 & 4.50 & 4.10 & 3.53 \\
\hline 47. & $\mathbf{T}_{4} \mathbf{V}_{11}$ & 5.03 & 4.37 & 3.87 & 3.57 \\
\hline 48. & $\mathbf{T}_{4} \mathbf{V}_{12}$ & 5.47 & 4.83 & 4.27 & 3.67 \\
\hline 49. & $\mathbf{T}_{5} \mathbf{V}_{1}$ & 4.13 & 3.63 & 3.37 & 3.87 \\
\hline 50. & $\mathbf{T}_{5} \mathbf{V}_{2}$ & 4.93 & 4.33 & 3.80 & 4.20 \\
\hline 51. & $\mathbf{T}_{5} \mathbf{V}_{3}$ & 5.27 & 4.67 & 4.23 & 4.87 \\
\hline 52. & $\mathbf{T}_{5} \mathbf{V}_{4}$ & 5.33 & 4.37 & 3.83 & 4.50 \\
\hline 53. & $\mathbf{T}_{5} \mathbf{V}_{5}$ & 6.67 & 5.90 & 5.27 & 4.57 \\
\hline 54. & $\mathbf{T}_{5} \mathbf{V}_{6}$ & 6.73 & 6.33 & 6.27 & 4.47 \\
\hline 55. & $\mathbf{T}_{5} \mathbf{V}_{7}$ & 5.67 & 5.00 & 4.23 & 4.30 \\
\hline 56. & $\mathbf{T}_{5} \mathbf{V}_{8}$ & 5.67 & 4.60 & 3.93 & 4.43 \\
\hline 57. & $\mathbf{T}_{5} \mathbf{V}_{9}$ & 6.53 & 5.53 & 4.87 & 4.43 \\
\hline 58. & $\mathbf{T}_{5} \mathbf{V}_{10}$ & 5.70 & 4.80 & 4.23 & 4.80 \\
\hline 59. & $\mathbf{T}_{5} \mathbf{V}_{11}$ & 5.70 & 4.83 & 4.17 & 3.47 \\
\hline 60. & $T_{5} V_{12}$ & 6.67 & 5.83 & 5.33 & 4.67 \\
\hline \multirow{2}{*}{\multicolumn{2}{|c|}{$\begin{array}{l}\operatorname{SEm}( \pm) \\
\operatorname{LSD}(0.05)\end{array}$}} & 0.196 & 0.152 & 0.132 & 0.126 \\
\hline & & 0.549 & 0.426 & 0.371 & 0.353 \\
\hline
\end{tabular}

Note: $\mathrm{T}_{1}=$ Aluminium foil, $\mathrm{T}_{2}=$ Cloth bag, $\mathrm{T}_{3}=$ Polythene packet, $\mathrm{T}_{4}=$ Brown paper packet, $\mathrm{T}_{5}=$ Freeze, $\mathrm{V}_{1}=$ Bidhan Marigold-1, $\mathrm{V}_{2}=$ Bidhan Basanti, $\mathrm{V}_{3}=\mathrm{BM}-3, \mathrm{~V}_{4}=\mathrm{BM}-4, \mathrm{~V}_{5}=\mathrm{BM}-5, \mathrm{~V}_{6}=\mathrm{BM}-6, \mathrm{~V}_{7}=$ Arka Bangara- $1, \mathrm{~V}_{8}=$ Arka Bangara- $2, \mathrm{~V}_{9}=$ Pusa Narangi, $\mathrm{V}_{10}=$ Yellow Single, $\mathrm{V}_{11}=$ Orange Single, $\mathrm{V}_{12}=$ Red Fresh Inka 
Table.4 Seedling shoot length $(\mathrm{cm})$ of different Marigold genotypes at three, six, nine and twelve months after storage

\begin{tabular}{|c|c|c|c|c|c|}
\hline \multirow{2}{*}{$\begin{array}{l}\text { Sl. } \\
\text { No. }\end{array}$} & \multirow{2}{*}{$\begin{array}{c}\text { Treatment } \\
\text { X } \\
\text { Genotype }\end{array}$} & \multicolumn{4}{|c|}{ Seedling shoot length $(\mathrm{cm})$} \\
\hline & & Three month & Six month & Nine month & Twelve month \\
\hline 1. & $\mathbf{T}_{1} \mathbf{V}_{1}$ & 3.97 & 3.37 & 3.17 & 3.17 \\
\hline 2. & $\mathbf{T}_{1} \mathbf{V}_{2}$ & 3.67 & 3.33 & 3.27 & 3.17 \\
\hline 3. & $\mathbf{T}_{1} \mathbf{V}_{3}$ & 3.97 & 3.67 & 3.40 & 3.27 \\
\hline 4. & $\mathbf{T}_{1} \mathbf{V}_{4}$ & 4.00 & 3.43 & 3.10 & 3.00 \\
\hline 5. & $\mathbf{T}_{1} \mathbf{V}_{5}$ & 3.80 & 3.40 & 3.23 & 3.00 \\
\hline 6. & $T_{1} \mathbf{V}_{6}$ & 3.40 & 3.20 & 3.20 & 3.03 \\
\hline 7. & $\mathbf{T}_{1} \mathbf{V}_{7}$ & 3.57 & 3.27 & 3.17 & 3.20 \\
\hline 8. & $\mathbf{T}_{1} \mathbf{V}_{8}$ & 3.33 & 3.10 & 3.07 & 3.03 \\
\hline 9. & $\mathbf{T}_{1} \mathbf{V}_{9}$ & 3.77 & 3.20 & 3.03 & 2.97 \\
\hline 10. & $T_{1} V_{10}$ & 3.37 & 3.23 & 3.23 & 3.13 \\
\hline 11. & $T_{1} V_{11}$ & 3.07 & 2.90 & 2.87 & 2.80 \\
\hline 12. & $T_{1} V_{12}$ & 3.87 & 3.43 & 3.33 & 3.17 \\
\hline 13. & $\mathbf{T}_{2} \mathbf{V}_{1}$ & 3.33 & 3.23 & 3.20 & 3.17 \\
\hline 14. & $\mathbf{T}_{2} \mathbf{V}_{2}$ & 3.67 & 3.27 & 3.23 & 3.23 \\
\hline 15. & $\mathbf{T}_{2} \mathbf{V}_{3}$ & 3.73 & 3.23 & 3.07 & 3.03 \\
\hline 16. & $\mathbf{T}_{2} \mathbf{V}_{4}$ & 3.73 & 3.10 & 3.00 & 2.97 \\
\hline 17. & $\mathbf{T}_{2} \mathbf{V}_{5}$ & 3.20 & 3.10 & 3.10 & 3.00 \\
\hline 18. & $\mathbf{T}_{2} \mathbf{V}_{6}$ & 3.43 & 3.20 & 3.23 & 3.20 \\
\hline 19. & $\mathbf{T}_{2} \mathbf{V}_{7}$ & 3.60 & 3.27 & 3.03 & 3.07 \\
\hline 20. & $\mathbf{T}_{2} \mathbf{V}_{8}$ & 3.37 & 3.30 & 3.23 & 3.13 \\
\hline 21. & $\mathbf{T}_{2} \mathbf{V}_{9}$ & 3.57 & 3.27 & 3.13 & 3.10 \\
\hline 22. & $\mathbf{T}_{2} \mathbf{V}_{10}$ & 3.53 & 3.30 & 3.30 & 3.20 \\
\hline 23. & $\mathbf{T}_{2} \mathbf{V}_{11}$ & 3.47 & 3.33 & 3.10 & 3.07 \\
\hline 24. & $\mathbf{T}_{2} \mathbf{V}_{12}$ & 3.97 & 3.63 & 3.23 & 3.20 \\
\hline 25. & $\mathbf{T}_{3} \mathbf{V}_{1}$ & 3.37 & 3.30 & 3.10 & 3.03 \\
\hline 26. & $\mathbf{T}_{3} \mathbf{V}_{2}$ & 3.63 & 3.30 & 3.27 & 3.13 \\
\hline 27. & $\mathbf{T}_{3} \mathbf{V}_{\mathbf{3}}$ & 3.53 & 3.23 & 3.17 & 3.03 \\
\hline 28. & $\mathbf{T}_{3} \mathbf{V}_{4}$ & 3.27 & 3.27 & 3.13 & 3.03 \\
\hline 29. & $\mathbf{T}_{3} \mathbf{V}_{5}$ & 3.57 & 3.23 & 3.07 & 3.03 \\
\hline 30. & $\mathbf{T}_{3} \mathbf{V}_{6}$ & 3.33 & 3.13 & 3.20 & 3.13 \\
\hline 31. & $\mathbf{T}_{3} \mathbf{V}_{7}$ & 3.47 & 3.23 & 3.30 & 3.23 \\
\hline 32. & $\mathbf{T}_{3} \mathbf{V}_{8}$ & 3.33 & 3.13 & 3.17 & 3.00 \\
\hline 33. & $\mathbf{T}_{3} \mathbf{V}_{9}$ & 3.53 & 3.23 & 3.10 & 3.13 \\
\hline 34. & $T_{3} V_{10}$ & 4.17 & 3.83 & 3.53 & 3.27 \\
\hline
\end{tabular}




\begin{tabular}{|c|c|c|c|c|c|}
\hline 35. & $\mathbf{T}_{3} \mathbf{V}_{11}$ & 3.47 & 3.33 & 3.13 & 3.07 \\
\hline 36. & $T_{3} V_{12}$ & 4.23 & 3.73 & 3.43 & 3.10 \\
\hline 37. & $\mathbf{T}_{4} \mathbf{V}_{1}$ & 3.27 & 3.23 & 3.13 & 3.07 \\
\hline 38. & $\mathbf{T}_{4} \mathbf{V}_{2}$ & 3.37 & 3.37 & 3.23 & 3.27 \\
\hline 39. & $\mathbf{T}_{4} \mathbf{V}_{\mathbf{3}}$ & 3.10 & 3.03 & 3.03 & 2.97 \\
\hline 40. & $\mathbf{T}_{4} \mathbf{V}_{4}$ & 3.40 & 3.23 & 3.20 & 3.03 \\
\hline 41. & $\mathbf{T}_{4} \mathbf{V}_{5}$ & 3.63 & 3.20 & 3.07 & 3.03 \\
\hline 42. & $\mathbf{T}_{4} \mathbf{V}_{6}$ & 3.23 & 3.10 & 3.10 & 2.97 \\
\hline 43. & $\mathbf{T}_{4} \mathbf{V}_{7}$ & 3.47 & 3.37 & 3.23 & 3.27 \\
\hline 44. & $\mathbf{T}_{4} \mathbf{V}_{8}$ & 3.67 & 3.27 & 3.07 & 3.03 \\
\hline 45. & $\mathbf{T}_{4} \mathbf{V}_{9}$ & 4.03 & 3.60 & 3.43 & 3.33 \\
\hline 46. & $\mathbf{T}_{4} \mathbf{V}_{10}$ & 3.37 & 3.30 & 3.13 & 3.03 \\
\hline 47. & $\mathbf{T}_{4} \mathbf{V}_{11}$ & 3.27 & 3.17 & 3.07 & 3.00 \\
\hline 48. & $\mathbf{T}_{4} \mathbf{V}_{12}$ & 4.27 & 3.93 & 3.57 & 3.43 \\
\hline 49. & $\mathbf{T}_{5} \mathbf{V}_{1}$ & 3.33 & 3.33 & 3.27 & 3.07 \\
\hline 50. & $\mathbf{T}_{5} \mathbf{V}_{2}$ & 4.03 & 3.63 & 3.27 & 3.10 \\
\hline 51. & $\mathbf{T}_{5} \mathbf{V}_{3}$ & 4.03 & 3.70 & 3.20 & 3.03 \\
\hline 52. & $\mathbf{T}_{5} \mathbf{V}_{4}$ & 3.50 & 3.20 & 3.17 & 2.87 \\
\hline 53. & $\mathbf{T}_{5} \mathbf{V}_{5}$ & 3.43 & 3.23 & 3.13 & 3.13 \\
\hline 54. & $\mathbf{T}_{5} \mathbf{V}_{6}$ & 3.90 & 3.47 & 3.20 & 3.07 \\
\hline 55. & $\mathbf{T}_{5} \mathbf{V}_{7}$ & 4.13 & 3.73 & 3.37 & 3.07 \\
\hline 56. & $\mathbf{T}_{5} \mathbf{V}_{8}$ & 3.93 & 3.43 & 3.17 & 2.97 \\
\hline 57. & $\mathbf{T}_{5} \mathbf{V}_{9}$ & 3.93 & 3.47 & 3.03 & 3.07 \\
\hline 58. & $\mathbf{T}_{5} \mathbf{V}_{10}$ & 3.87 & 3.53 & 3.30 & 3.27 \\
\hline 59. & $\mathbf{T}_{5} \mathbf{V}_{11}$ & 4.00 & 3.43 & 3.30 & 3.17 \\
\hline 60. & $\mathbf{T}_{5} \mathbf{V}_{12}$ & 4.50 & 3.90 & 3.37 & 3.33 \\
\hline \multirow{2}{*}{\multicolumn{2}{|c|}{$\begin{array}{l}\operatorname{SEm}( \pm) \\
\operatorname{LSD}(0.05)\end{array}$}} & 0.160 & 0.106 & 0.089 & 0.080 \\
\hline & & 0.450 & 0.297 & 0.250 & 0.225 \\
\hline
\end{tabular}

Note: $\mathrm{T}_{1}=$ Aluminium foil, $\mathrm{T}_{2}=$ Cloth bag, $\mathrm{T}_{3}=$ Polythene packet, $\mathrm{T}_{4}=$ Brown paper packet, $\mathrm{T}_{5}=$ Freeze, $\mathrm{V}_{1}=$ Bidhan Marigold-1, $\mathrm{V}_{2}=$ Bidhan Basanti, $\mathrm{V}_{3}=\mathrm{BM}-3, \mathrm{~V}_{4}=\mathrm{BM}-4, \mathrm{~V}_{5}=\mathrm{BM}-5, \mathrm{~V}_{6}=\mathrm{BM}-6, \mathrm{~V}_{7}=$ Arka Bangara- $1, \mathrm{~V}_{8}=$ Arka Bangara- $2, \mathrm{~V}_{9}=$ Pusa Narangi, $\mathrm{V}_{10}=$ Yellow Single, $\mathrm{V}_{11}=$ Orange Single, $\mathrm{V}_{12}=$ Red Fresh Inka 
Table.5 Vigour index of different Marigold genotypes at three, six, nine and twelve months after storage

\begin{tabular}{|c|c|c|c|c|c|}
\hline \multirow{2}{*}{$\begin{array}{l}\text { Sl. } \\
\text { No. }\end{array}$} & \multirow{2}{*}{$\begin{array}{l}\text { Treatment } \\
\text { X Genotype }\end{array}$} & \multicolumn{4}{|c|}{ Vigour index } \\
\hline & & Three month & Six month & Nine month & Twelve month \\
\hline 1. & $\mathbf{T}_{1} \mathbf{V}_{1}$ & 570.81 & 425.97 & 329.26 & 278.50 \\
\hline 2. & $\mathbf{T}_{1} \mathbf{V}_{2}$ & 589.00 & 440.91 & 357.32 & 289.13 \\
\hline 3. & $\mathbf{T}_{1} \mathbf{V}_{\mathbf{3}}$ & 621.19 & 507.45 & 385.57 & 291.29 \\
\hline 4. & $\mathbf{T}_{1} \mathbf{V}_{4}$ & 616.82 & 454.19 & 335.01 & 279.69 \\
\hline 5. & $T_{1} \mathbf{V}_{5}$ & 585.07 & 466.78 & 355.78 & 286.11 \\
\hline 6. & $T_{1} V_{6}$ & 534.10 & 445.26 & 352.41 & 285.88 \\
\hline 7. & $\mathbf{T}_{1} \mathbf{V}_{7}$ & 635.19 & 495.82 & 382.63 & 299.66 \\
\hline 8. & $\mathbf{T}_{1} \mathbf{V}_{8}$ & 637.76 & 462.60 & 357.29 & 290.46 \\
\hline 9. & $\mathbf{T}_{1} \mathbf{V}_{9}$ & 712.53 & 570.57 & 444.64 & 352.58 \\
\hline 10. & $T_{1} V_{10}$ & 538.05 & 460.00 & 360.42 & 279.50 \\
\hline 11. & $T_{1} V_{11}$ & 548.93 & 423.93 & 329.65 & 262.84 \\
\hline 12. & $T_{1} V_{12}$ & 628.37 & 512.18 & 399.63 & 309.21 \\
\hline 13. & $\mathbf{T}_{2} \mathbf{V}_{1}$ & 645.29 & 499.25 & 375.17 & 284.65 \\
\hline 14. & $\mathbf{T}_{2} \mathbf{V}_{2}$ & 661.25 & 500.00 & 387.65 & 349.09 \\
\hline 15. & $\mathbf{T}_{2} \mathbf{V}_{3}$ & 756.94 & 573.61 & 436.84 & 338.13 \\
\hline 16. & $\mathbf{T}_{2} \mathbf{V}_{4}$ & 761.19 & 585.89 & 449.98 & 334.98 \\
\hline 17. & $\mathbf{T}_{2} \mathbf{V}_{5}$ & 710.30 & 587.29 & 459.13 & 366.08 \\
\hline 18. & $\mathbf{T}_{2} \mathbf{V}_{6}$ & 769.30 & 610.60 & 463.52 & 362.10 \\
\hline 19. & $\mathbf{T}_{2} \mathbf{V}_{7}$ & 676.38 & 553.20 & 419.06 & 336.55 \\
\hline 20. & $\mathbf{T}_{2} \mathbf{V}_{8}$ & 676.60 & 530.56 & 409.37 & 319.74 \\
\hline 21. & $\mathbf{T}_{2} \mathbf{V}_{9}$ & 745.22 & 595.94 & 458.71 & 374.79 \\
\hline 22. & $\mathbf{T}_{2} \mathbf{V}_{10}$ & 625.98 & 485.67 & 374.18 & 293.48 \\
\hline 23. & $\mathbf{T}_{2} \mathbf{V}_{11}$ & 466.87 & 402.53 & 316.10 & 261.81 \\
\hline 24. & $\mathbf{T}_{2} \mathbf{V}_{12}$ & 552.69 & 448.09 & 345.03 & 289.32 \\
\hline 25. & $\mathbf{T}_{3} \mathbf{V}_{1}$ & 626.62 & 493.94 & 357.47 & 289.28 \\
\hline 26. & $\mathbf{T}_{3} \mathbf{V}_{2}$ & 675.38 & 503.30 & 430.81 & 296.42 \\
\hline 27. & $\mathbf{T}_{3} \mathbf{V}_{3}$ & 688.56 & 575.95 & 429.40 & 331.68 \\
\hline 28. & $\mathbf{T}_{3} \mathbf{V}_{4}$ & 632.29 & 551.56 & 420.87 & 303.50 \\
\hline 29. & $\mathbf{T}_{3} \mathbf{V}_{5}$ & 728.30 & 591.40 & 455.04 & 380.59 \\
\hline 30. & $\mathbf{T}_{3} \mathbf{V}_{6}$ & 684.86 & 558.94 & 437.77 & 428.93 \\
\hline 31. & $\mathbf{T}_{3} \mathbf{V}_{7}$ & 728.23 & 597.71 & 463.99 & 318.57 \\
\hline 32. & $\mathbf{T}_{3} \mathbf{V}_{8}$ & 686.24 & 560.28 & 442.08 & 314.99 \\
\hline 33. & $\mathbf{T}_{3} \mathbf{V}_{9}$ & 709.01 & 552.44 & 425.16 & 360.76 \\
\hline 34. & $T_{3} V_{10}$ & 676.67 & 533.62 & 401.18 & 315.71 \\
\hline 35. & $T_{3} V_{11}$ & 582.06 & 494.32 & 368.90 & 309.10 \\
\hline 36. & $T_{3} V_{12}$ & 745.24 & 581.82 & 445.62 & 399.04 \\
\hline 37. & $\mathbf{T}_{4} \mathbf{V}_{1}$ & 621.97 & 502.16 & 393.22 & 320.61 \\
\hline
\end{tabular}




\begin{tabular}{|c|c|c|c|c|c|}
\hline 38. & $\mathbf{T}_{4} \mathbf{V}_{2}$ & 690.44 & 546.94 & 412.74 & 325.16 \\
\hline 39. & $\mathbf{T}_{4} \mathbf{V}_{3}$ & 649.91 & 548.09 & 433.89 & 345.36 \\
\hline 40. & $\mathbf{T}_{4} \mathbf{V}_{4}$ & 691.92 & 557.91 & 434.06 & 335.86 \\
\hline 41. & $\mathbf{T}_{4} \mathbf{V}_{5}$ & 735.23 & 573.65 & 422.45 & 333.79 \\
\hline 42. & $\mathbf{T}_{4} \mathbf{V}_{6}$ & 677.87 & 539.30 & 414.94 & 315.83 \\
\hline 43. & $\mathbf{T}_{4} \mathbf{V}_{7}$ & 667.62 & 456.84 & 339.67 & 292.67 \\
\hline 44. & $\mathbf{T}_{4} \mathbf{V}_{8}$ & 616.10 & 450.28 & 348.00 & 285.92 \\
\hline 45. & $\mathbf{T}_{4} \mathbf{V}_{9}$ & 810.47 & 648.92 & 519.15 & 406.99 \\
\hline 46. & $T_{4} V_{10}$ & 593.21 & 475.69 & 360.73 & 275.29 \\
\hline 47. & $T_{4} V_{11}$ & 588.39 & 471.28 & 357.54 & 285.47 \\
\hline 48. & $T_{4} V_{12}$ & 723.61 & 579.06 & 433.27 & 336.16 \\
\hline 49. & $\mathbf{T}_{5} \mathbf{V}_{1}$ & 550.81 & 451.04 & 356.32 & 309.23 \\
\hline 50. & $\mathbf{T}_{5} \mathbf{V}_{2}$ & 679.53 & 532.41 & 394.41 & 346.20 \\
\hline 51. & $\mathbf{T}_{5} \mathbf{V}_{\mathbf{3}}$ & 699.54 & 560.29 & 417.43 & 378.17 \\
\hline 52. & $\mathbf{T}_{5} \mathbf{V}_{4}$ & 660.41 & 503.20 & 388.23 & 351.42 \\
\hline 53. & $\mathbf{T}_{5} \mathbf{V}_{5}$ & 763.15 & 614.64 & 473.22 & 362.46 \\
\hline 54. & $\mathbf{T}_{5} \mathbf{V}_{6}$ & 801.54 & 657.09 & 530.42 & 360.18 \\
\hline 55. & $\mathbf{T}_{5} \mathbf{V}_{7}$ & 732.22 & 579.71 & 421.98 & 355.34 \\
\hline 56. & $\mathbf{T}_{5} \mathbf{V}_{8}$ & 726.33 & 542.01 & 398.54 & 355.39 \\
\hline 57. & $\mathbf{T}_{5} \mathbf{V}_{9}$ & 803.76 & 619.15 & 456.99 & 372.70 \\
\hline 58. & $T_{5} V_{10}$ & 684.48 & 526.42 & 394.14 & 350.40 \\
\hline 59. & $T_{5} V_{11}$ & 701.75 & 529.64 & 396.56 & 292.30 \\
\hline 60. & $T_{5} V_{12}$ & 845.57 & 657.06 & 494.25 & 376.15 \\
\hline \multirow{2}{*}{\multicolumn{2}{|c|}{$\begin{array}{l}\operatorname{SEm}( \pm) \\
\operatorname{LSD}(0.05)\end{array}$}} & 7.405 & 6.980 & 5.912 & 6.033 \\
\hline & & 20.762 & 19.568 & 16.574 & 16.915 \\
\hline
\end{tabular}

Note: $\mathrm{T}_{1}=$ Aluminium foil, $\mathrm{T}_{2}=$ Cloth bag, $\mathrm{T}_{3}=$ Polythene packet, $\mathrm{T}_{4}=$ Brown paper packet, $\mathrm{T}_{5}=$ Freeze, $\mathrm{V}_{1}=$ Bidhan Marigold-1, $\mathrm{V}_{2}=$ Bidhan Basanti, $\mathrm{V}_{3}=\mathrm{BM}-3, \mathrm{~V}_{4}=\mathrm{BM}-4, \mathrm{~V}_{5}=\mathrm{BM}-5, \mathrm{~V}_{6}=\mathrm{BM}-6, \mathrm{~V}_{7}=$ Arka Bangara- $1, \mathrm{~V}_{8}=$ Arka Bangara- $2, \mathrm{~V}_{9}=$ Pusa Narangi, $\mathrm{V}_{10}=$ Yellow Single, $\mathrm{V}_{11}=$ Orange Single, $\mathrm{V}_{12}=$ Red Fresh Inka 

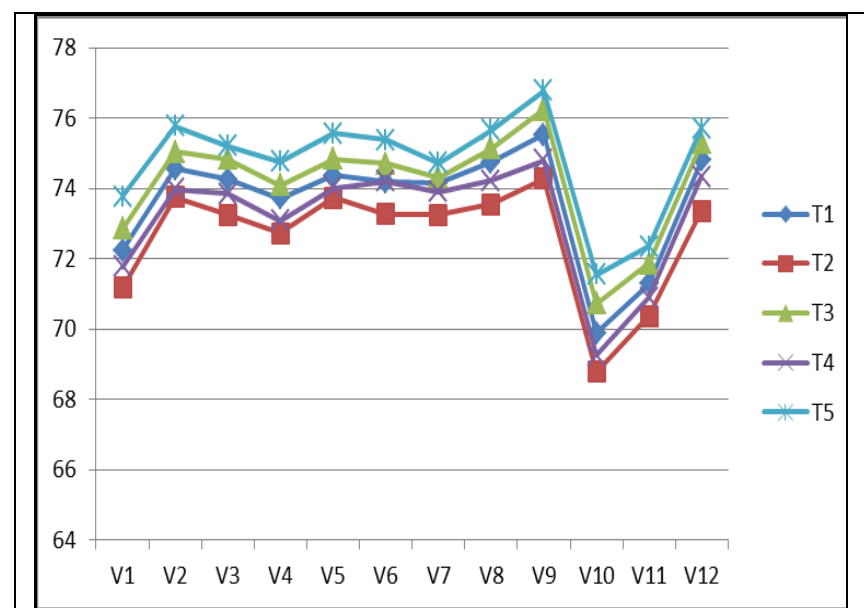

Fig.1 Germination \% at three months after

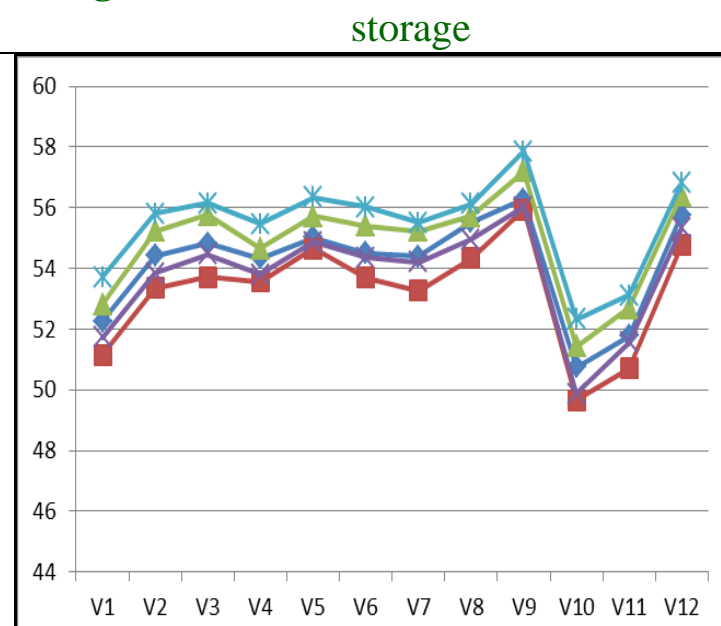

Fig.3 Germination \% at nine months after storage

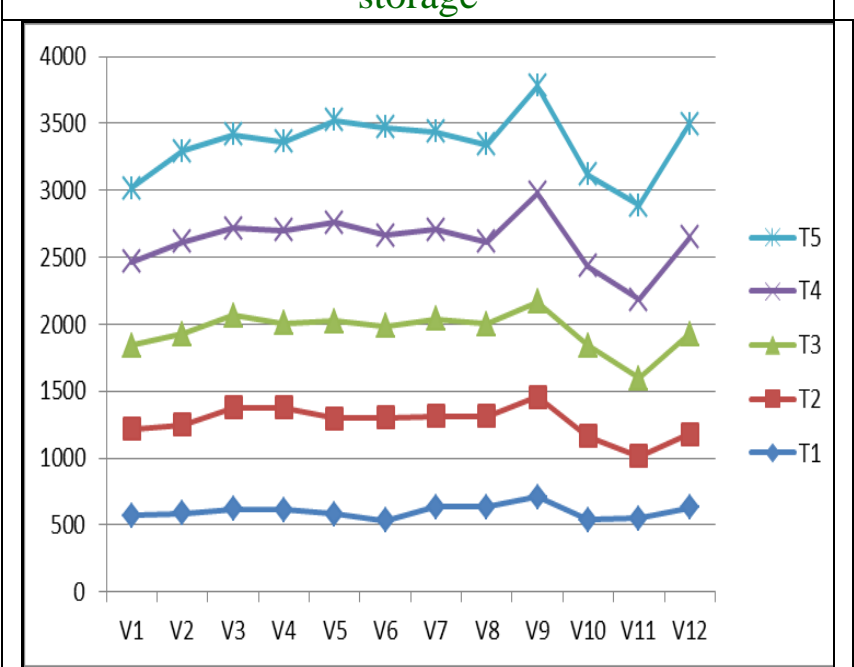

Fig.5 Vigour index at three months after storage

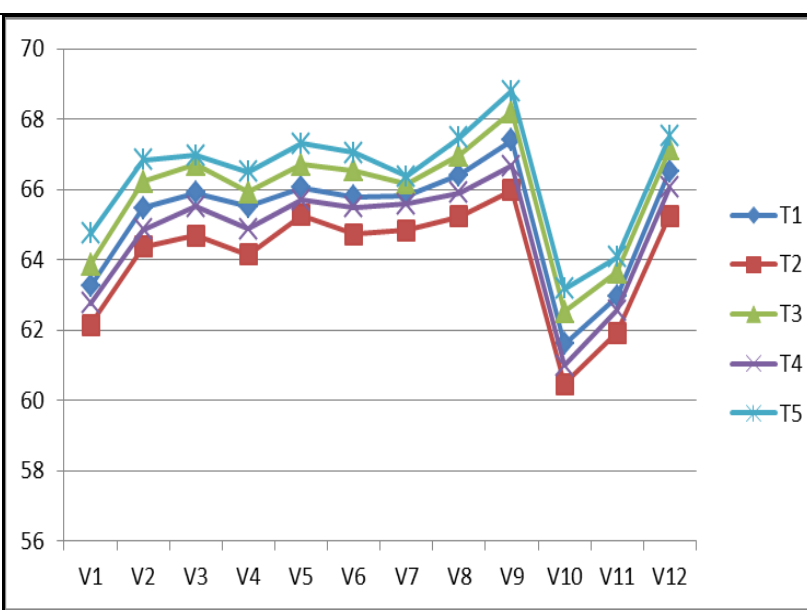

Fig.2 Germination \% at six months after storage

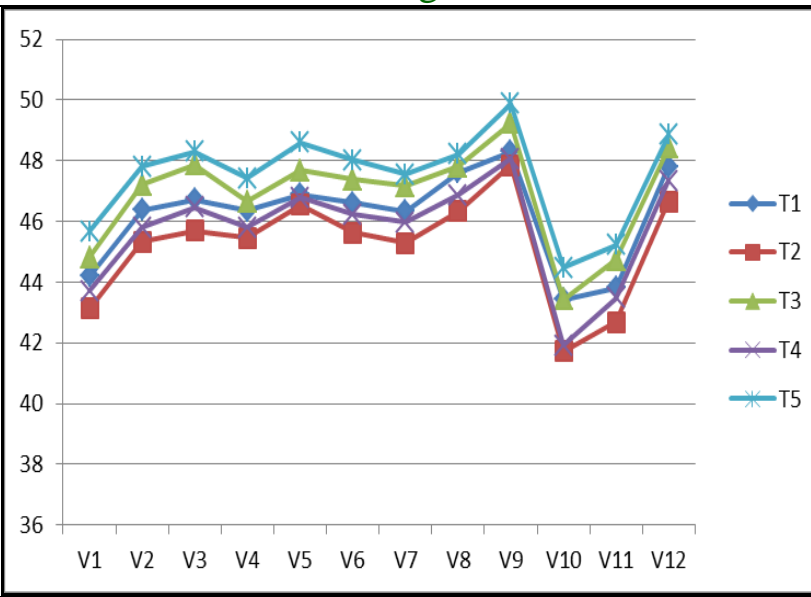

Fig.4 Germination \% at twelve months after storage

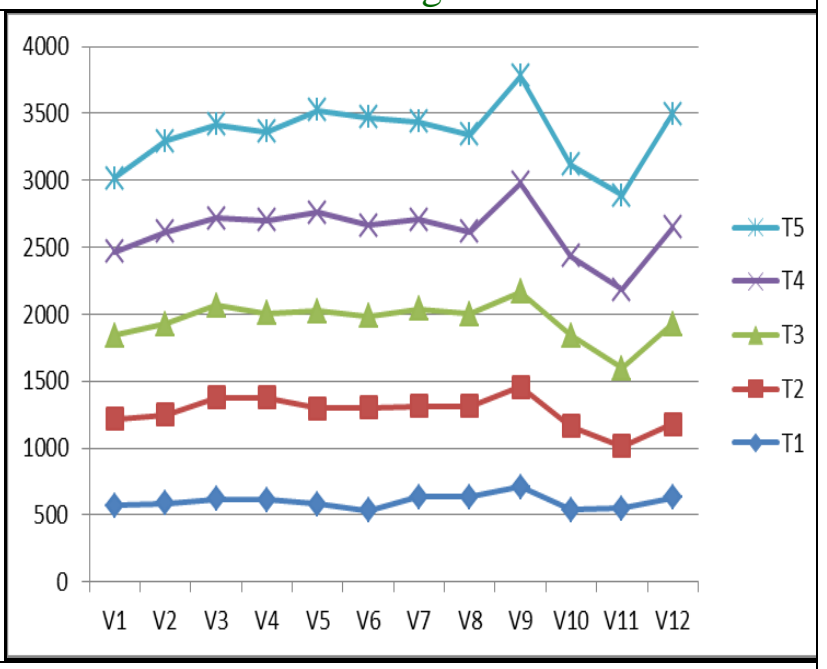

Fig.6 Vigour index at six months after storage 


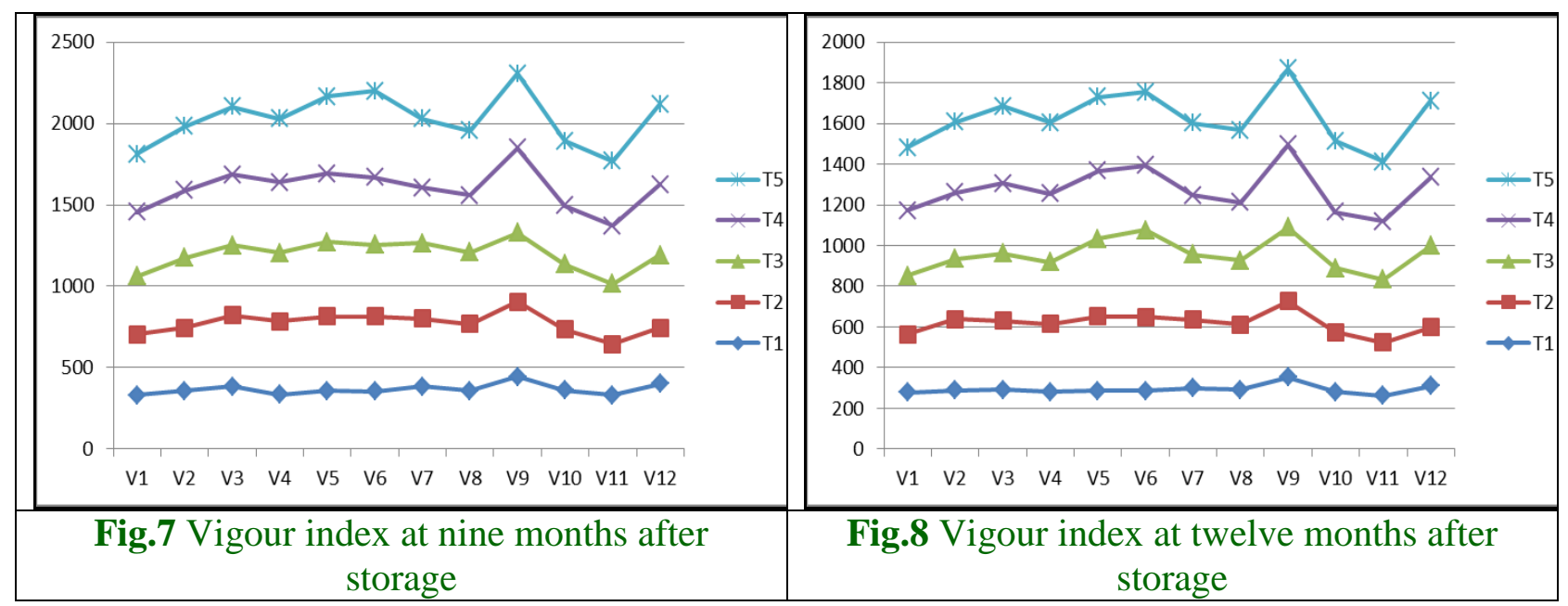

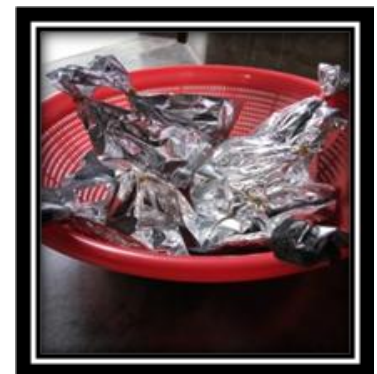

Aluminium foil

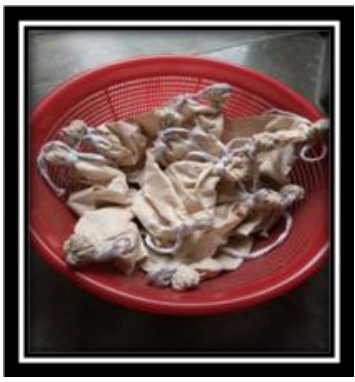

Cloth bag

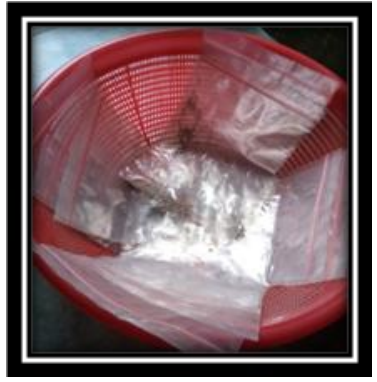

Polythene packet Brown paper packet

Fig.9 Marigold seeds stored in different storage conditions
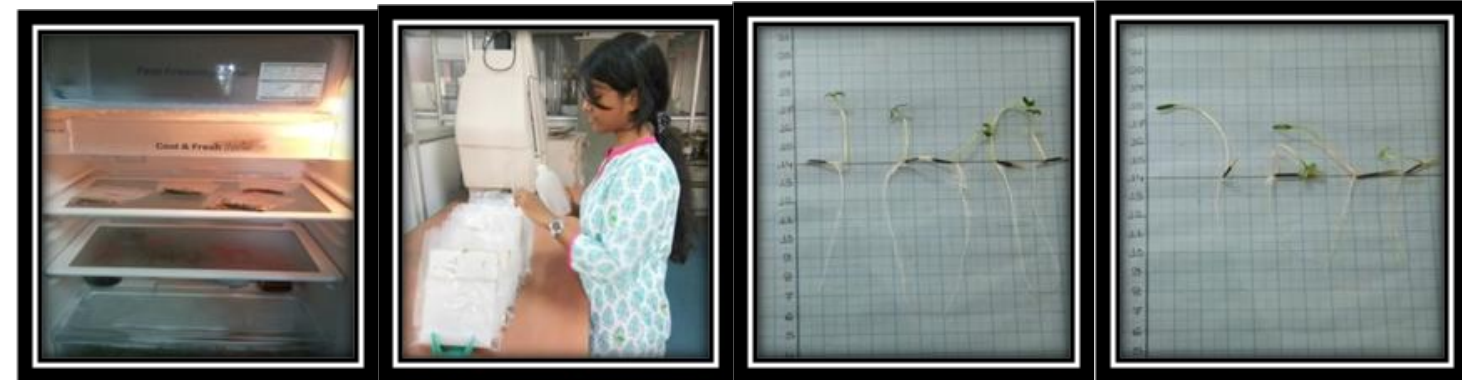

Refrigerator Preparing glass plates Pusa Narangi (Aluminium foil) Pusa Narangi (Cloth bag)
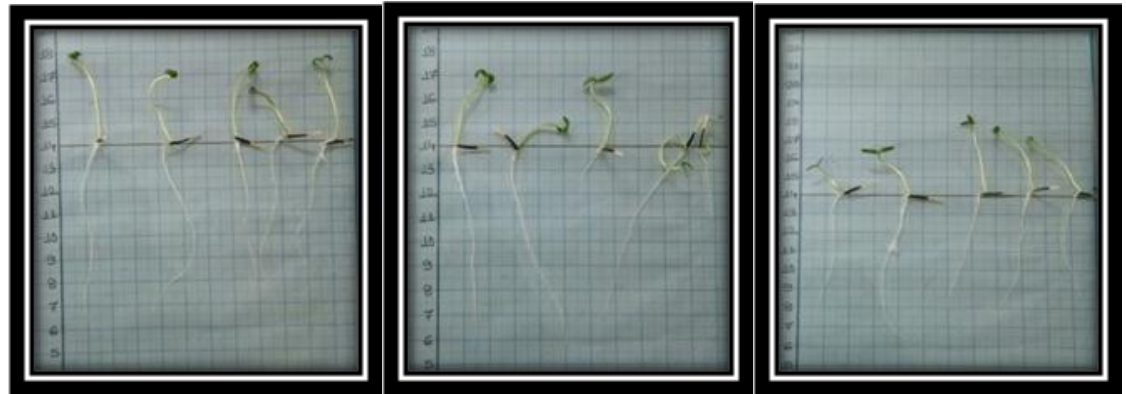

Pusa Narangi (Polythene) Pusa Narangi (Brown paper) Pusa Narangi (Refrigerator)

Fig.10 Evaluation of seedling root and shoot length through glass plate method 
Vigour index at three, six, nine and twelve months after storage

Refrigerator storage influenced in the best way, on an average for determining highest vigour index of stored seeds for all storage durations followed by $\mathrm{T}_{3}$ (Polythene packet of 300 gauge thickness), excepting at nine months of storage wherein cloth bag and polythene packet storage influenced this parameter in statistically similar manner and lowest vigour index could be noticed after storage in aluminium foil.

Critical consideration of interaction between genotypes and storage conditions indicated different scenario at different storage durations of the study: refrigerator storage was preferred by most of the genotypes at three months of storage for determination of vigour index excepting $V_{3}$ and $V_{4}$ for which cloth bag storage was found to be preferred best, while it was of significantly lowest magnitude for aluminium foil storage for seven genotypes, it was three for cloth bag storage and one each for polythene and brown paper packet storage; best preference towards refrigerated storage could be noticed for eight genotypes after six months of storage, five genotypes at nine months after storage and seven genotypes at twelve months after storage; sequential performance towards storage conditions became progressively erratic with the advancement in storage duration when determined vigour index was considered.

Therefore unique response of individual genotypes could also be noticed for this important seed quality parameter, which may be due to the determining procedure of vigour index - germination percentage $\mathrm{x}$ seedling length for the present case, both varied tremendously with the advancement of storage duration.
Seed storage of all the genotypes failed to attain prescribed germination $(70 \%)$ as per MSCS of Marigold beyond three months after storage irrespective of the storage conditions; hence, different conditions under consideration may not be recommended for storing of Marigold seeds beyond three months. Joint consideration of germination potential and vigour index indicate that seed storage within refrigerator as well as in 300 gauge polythene packet may be recommended for all the genotypes studied upto three months for maintenance of its higher quality.

\section{Acknowledgement}

The authors thankfully acknowledge the support and facilities provided by the seed testing laboratory, Department of Seed science and Technology, Bidhan Chandra KrishiViswavidyalaya, Mohanpur, Nadia, West Bengal, India.

\section{References}

Chakraborty, A., Bordolui, S.K., Mahato, M. K., Sadhukhan, R. and Sri Veda, D.J.M.S.N.K. 2019. Variation in seed production potential of China aster genotypes in the New Alluvial Zone of West Bengal. J. Crop and Weed. 15(1): 201-204.

Chakraborty, A., Bordolui, S.K., Nandi, D. and Mahato, M. K. 2020. Seed Deterioration Pattern of Some China Aster During Storage. Int.J.Curr.Microbiol.App.Sci. $\quad 9(03)$ : 1499-1506.

Gajanana, T. M. and Sudha, M. 2006. Production and Marketing Research on Traditional Flowers. Advances in Ornamental Horticulture.6: 19-23.

Gawle, S. K., Chaturvedi, M. K. and Yadaw, K. N. (2012). Adoption pattern of improved marigold production technologies by the farmers in 
Bilashpur district of Chhattisgarh. Agriculture Update. 7(3/4): 323-329.

Kumar, T. P., Asha, A. M., Maruthi, J. B. and Vishwanath, K. 2014. Influence of seed treatment chemicals and containers on seed quality of marigold during storage. The Bioscan. 9(3): 937-942.

Kumar, T. P.,Radha, B. N.,Vishwanath, K.,Rao, G. G. E. andHullur, N. 2014. Seed storage quality as influenced by forms of seed and containers during storage in marigold (Tagetes erecta). International Journal of Agricultural Sciences. 10(2): 700-706.

Kumari, P., Bordolui, S.K. and Sadhukhan, R. 2017. Seed quality deterioration of some winter flowers during storage. Journal of Crop and Weed, 13(1):164169.

Mahato, M. K., Bordolui, S.K., Chakraborty, A., Das, R. and Muru, K. 2019. Seed quality deterioration of some China aster genotypes during storage. Journal of Emerging Technologies and Innovative Research (JETIR). 6(2):467473.

Saxena, M. and Gandhi, C. P.2015.National Horticulture Board. Indian Horticulture Database. pp. 286.

Selvaraju, P., Jacqueline, A. and Selvaraj. 1999. Effect of seed treatments and storage containers on viability and vigour of seeds in marigold (Tagetes erecta L.). South Indian Horticulture. 47(1/6): 364-366.

Vasudev, H. S., Umashankar, N.,Devagiri, G. M., Krishnamurthy, R.,Raveendra, H. R. and Rajkumar, M. 2009. Effect of seed storage methods on seed quality of marigold (Tagetes erecta). Mysore Journal of Agricultural Sciences. 43(2): $239-245$.

\section{How to cite this article:}

Jui Ray and Sanjoy Kumar Bordolui. 2020. Seed Storing Potential of Some Marigold Genotypes during Storage. Int.J.Curr.Microbiol.App.Sci. 9(05): 2470-2486. doi: https://doi.org/10.20546/ijcmas.2020.905.283 